mTORC2 is
specifically
upregulated ...
under high $\mathrm{K}^{+}$
conditions and
increases the
activity and
abundance of
the renal outer
medulla $\mathrm{K}^{+}$
channel

$\Rightarrow$ RENAL PHYSIOLOGY \title{
mTORC2 controls potassium secretion
}

The mTOR complexes, mTORC1 and mTORC2, are involved in numerous renal diseases, but the function of mTORC2 in the kidney is poorly understood. A new study from Florian Grahammer, Tobias Huber and colleagues shows that mTORC2 is a critical regulator of distal tubular $\mathrm{K}^{+}$ secretion.

"Due to the lack of an mTORC2-specific inhibitor, we used a conditional mouse genetic approach to investigate mTORC2 function," explains Huber. "Deletion of the functionally essential subunit RICTOR specifically abrogated mTORC2 signalling in distal tubular cells," adds Grahammer.

Although previous studies using mTOR inhibitors had suggested that mTORC2 regulates the function of the epithelial sodium channel $\mathrm{ENaC}$, the researchers found that mTORC2-deficient mice adequately reduced $\mathrm{Na}^{+}$secretion in response to a low $-\mathrm{Na}^{+}$diet. "ENaC might not be a central component of mTORC2 signalling," suggests Huber.

When challenged with a high- $\mathrm{K}^{+}$diet, the mTORC2-deficient mice developed life-threatening hyperkalaemia despite a 10 -fold upregulation of aldosterone levels. "We found that mTORC2 is specifically upregulated in control animals under high $\mathrm{K}^{+}$conditions and increases the activity and abundance of the renal outer medulla $\mathrm{K}^{+}$ channel ROMK via phosphorylation of SGK1 and PKCa," says Grahammer.

"Given the importance of $\mathrm{K}^{+}$for body homeostasis, we identified mTORC2 as a central regulator of $\mathrm{K}^{+}$excretion, upstream of known transport pathways in the aldosteronesensitive nephron" concludes Huber.

The researchers now plan to elucidate the molecular mechanism by which increases in extracellular $\mathrm{K}^{+}$levels lead to upregulation of mTORC2 expression. They will also investigate if and how this $\mathrm{K}^{+}$regulatory axis is altered in animal models of chronic kidney disease and if increasing mTORC2 signalling modulates the $\mathrm{K}^{+}$balance in these models.

Andrea Aguilar

ORIGINAL ARTICLE(S) Grahammer, F. et al. mTORC2 critically regulates renal potassium handling.J. Clin. Invest. http://dx.doi.org/10.1172/JCl80304 (2016) 